\title{
A miniaturized hairpin resonator for the high selectivity of WLAN bandwidth
}

\author{
S. M. Kayser Azam, Muhammad I. Ibrahimy, S. M. A. Motakabber,
}

A. K. M. Zakir Hossain, Md. Shazzadul Islam

Department of Electrical and Computer Engineering, International Islamic University Malaysia, Malaysia

\begin{tabular}{l}
\hline \hline Article Info \\
\hline Article history: \\
Received Feb 1, 2019 \\
Revised Apr 3, 2019 \\
Accepted Apr 18, 2019 \\
\hline Keywords: \\
Bandpass filter \\
Hairpin resonator \\
High selectivity \\
Microstrip \\
WLAN
\end{tabular}

\begin{abstract}
In this article, a miniaturized hairpin resonator has been presented to introduce the high selectivity of Wireless Local Area Network (WLAN) bandwidth. In the construction of the hairpin resonator, short-circuited comb-lines are electrically coupled with the two longer edges of a rectangular-shaped loop. The hairpin resonator has been designed and fabricated with the Taconic TLX-8 substrate with a center-frequency at 2.45 GHz. The resonator exhibits a second order quasi-Chebyshev bandpass response. A low insertion loss has been found as $-0.36 \mathrm{~dB}$ with a minimum return loss as $-36.71 \mathrm{~dB}$. The filtering dimension of this hairpin resonator occupies a small area of $166.82 \mathrm{~mm}^{2}$. This hairpin resonator is highly selective for the bandpass applications of the entire WLAN bandwidth.
\end{abstract}

\section{Copyright (c) 2019 Institute of Advanced Engineering and Science.} All rights reserved.

\section{Corresponding Author:}

Muhammad I. Ibrahimy,

Department of Electrical and Computer Engineering,

International Islamic University Malaysia,

Kulliyah of Engineering, 53100 Jalan Gombak, Kuala Lumpur, Malaysia.

Email: ibrahimy@iium.edu.my

\section{INTRODUCTION}

For most of the systems in wireless communication, microstrip resonators are widely used as bandpass filters so that the undesired signals cannot interfere with the specified bandwith. Following the IEEE 802.11 standards, a bandwidth of $100 \mathrm{MHz}$ from $2.4 \mathrm{GHz}$ frequency to $2.5 \mathrm{GHz}$ frequency is assigned for WLAN and Industrial Scientific and Medical (ISM) applications. Despite the recent growth of monolithic microwave integrated circuits (MMICs) and ultra-wide band (UWB) technologies, planar resonators and filters keep their demands in the ISM and WLAN applications, especially, after the emerging trends of the internet of things (IoT) [1-4]. However, microstrip resonators designed as bandpass filters for ISM and WLAN applications still offer challenges to exhibit high selectivity of $100 \mathrm{MHz}$ bandwidth in the ISM and WLAN bands with a low insertion loss and a small chip area.

In recent, to ensure a low level of insertion loss, a hairpin resonator-based four-pole ellipticfunctioned bandpass filter has been intoduced [5]. In the hairpin resonator construction, some interdigital lines are employed for the miniaturization purpose. Nevertheless, the resonator in [5] has large fractional bandwidth that allows the out-band signal to interfere with the WLAN bandwidth. In fact, the center frequency selected for the resonator in [5] is exactly at the lower frequency of WLAN bandwith. As a result, the resonator in [5] has the limitations of poor selectivity in terms of WLAN frequencies. To obtain high selectivity, a four-pole bandpass filter with the K-inverter has been proposed in [6] for selecting the center frequency within the WLAN bandwidth. However, the filter exhibits $13.81 \%$ fractional bandwidth that allows the out-band signals to interfere with the WLAN bandwidth. Besides, the filter designed in [6] has a large dimension. 
To miniaturize the resonator, a fragmented loaded-resonator has been introduced in [7]. Despite the resonator ensures a small dimension, the fragmented design is cumbersome to implement. In fact, there is no clear-cut methodology of the fragmented loaded-resonator that can be implemented with different specifications. In addition, the resonator transmits the signal from the out-band since the center frequency is not selected at the middle of the WLAN bandwidth. To further miniaturize the resonators for the bandpass applications, defected ground structure (DGS) technique has been applied in [8]. The filter based on DGS in [8] occupies a very small area of $154 \mathrm{~mm}^{2}$. Yet, the filter based on DGS in [8] provides more insertion loss than that of the resonator in [7]. Meanwhile, the insertion loss is remarkable decreased to $-0.40 \mathrm{~dB}$ by using the ring resonator in [9] with the short-ended self-coupled technique but it requires a voluminous dimension. For achieving a compact dimension, a filter has been proposed in [10] by using the substrate integrated waveguide (SIW) technique along with the DGS. Despite the resonator covers a small area of $110.5 \mathrm{~mm}^{2}$, it offers a high insertion loss of $-2.30 \mathrm{~dB}$ that is a noticeable limitaion of the design. Whereas, in [11] a microstrip hairpin resonator has been presented with a precise selectivity of the center frequency. However, the resonator offers a bulky chip area with a large fractional bandwidth that is unable to eliminate the out-band signal for the WLAN bandwidth. Recently, filters with the microstrip stepped impedance resonator (SIR) technique [12], [13] have been proposed to overcome the drawbacks of the large fractional bandwidth, the high insertion loss and the bulky chip area. In [12], the filter offers a small chip area but with an inappropriate fractional bandwidth for attenuating out-band signals of WLAN bandwidth. Meanwhile in [13], a bandpass filter has been introduced that suffers from an unsatisfactory physical dimension and incompatible center frequency for tightly selecting the WLAN bandwidth. In recent, a frequency-dependent source-load coupling technique and the coupled line radial stubs-based technique [14], [15] are proposed. However, these filters yet have the drawbacks of loosely selecting the WLAN bandwidth of $100 \mathrm{MHz}$ by lowering the insertion loss and eliminating the out-band signal.

To deal with these problems, a miniaturized hairpin resonator with bandpass response has been introduced in this article. The resonator is designed at a center frequency of $2.45 \mathrm{GHz}$ which is exactly the middle frequency of WLAN bandwidth. The designed hairpin resnoator tightly selects the entire band of WLAN bandwidth with the lowest insertion loss of $-0.36 \mathrm{~dB}$ and a small area of $166.82 \mathrm{~mm}^{2}$.

\section{RESONATOR SYNTHESIS}

Prior to designing the hairpin resonator, a second-ordered Chebyshev ideal low pass filter prototype has been chosen for transforming it into the bandpass response with lossless lumped components by employing the conventional technique of filter design [16]. Element values have been selected for an ideal Chebyshev response with a $0.01 \mathrm{~dB}$ ripple. Hence, $g_{0}=1, g_{1}=0.4489, g_{2}=0.4078$ and $g_{3}=0.9085$ are selected for the second-ordered Chebyshev response. It is noteworthy, a $100 \mathrm{MHz}$ bandwidth is estimated for the $2.45 \mathrm{GHz}$ center frequency so that the entire WLAN bandwidth is covered. However, because the resonator is to be realized by the microstrip planar technology, the lossless lumped components have been translated into the microstrip lines by the characteristic impedance $\left(Z_{0}\right)$ technique [16, 17]. For the characteristic impedance technique, the dielectric substrate considered for the microstrip design has the effective dielectric permittivity $\left(\epsilon_{e}\right)$ by employing the equations below.

$$
\begin{aligned}
& \epsilon_{e}=\frac{\varepsilon_{r}+1}{2}+\frac{\varepsilon_{r}-1}{2} \frac{1}{\sqrt{1+12 d / w}} \\
& Z_{0}=\frac{60}{\sqrt{\epsilon_{e}}} \ln \left(\frac{8 d}{w}+\frac{w}{4 d}\right) \\
& Z_{0}=\frac{120 \pi}{\sqrt{\epsilon_{e}}\left[\frac{w}{d}+1.393+0.667 \ln \left(\frac{w}{d}+1.444\right)\right]}
\end{aligned}
$$

In these equations above, the width $(w)$ of a microstrip line, the thickness $(d)$ of the substrate and the dielectric permittivity $\left(\varepsilon_{r}\right)$ of the substrate material determine the characteristic impedance of the microstrip line. Equation (2) is valid for obtaining the characteristic impedance of any microstrip line while the ratio between $w$ and $d$ is below 1. Whereas, (3) is applicable when the same ratio is above 1.

Following the aforementioned technique, the Taconic TLX-8 substrate has been selected for designing the hairpin resonator. This substrate has the $\varepsilon_{r}$ of 2.55 and the tangent loss-factor of 0.0019 . Moreover, the Taconic TLX-8 substrate has a thickness of $0.5 \mathrm{~mm}$ excluding the copper thickness. Such a thin substrate layer makes it a good candidate for wearable and less-weighted applications. On the basis of the coupled-line theory [16], the proposed resonator has been designed at $2.45 \mathrm{GHz}$ center frequency. 
Figure 1 illustrates the construction of the proposed resonator. In Figure 1(a), a rectangular-shaped resonator has been coupled at the two larger stubs $\left(l_{2}\right.$ and $\left.l_{3}\right)$ with two comb-lines $\left(l_{1}\right.$ and $\left.l_{4}\right)$ in a way so that $l_{1}=l_{2}=l_{3}=l_{4}$. Therefore, a strong electric coupling has been achieved because of the mutual capacitance between every comb-line and the larger stub of the rectangular loop. At the complementary ends of each comb-line, microstrip tapered lines are connected to construct a short-circuited path to the ground plane via conductor. Consequently, each of the comb-lines is short-circuited by the stepped impedance technique. Meanwhile, each of the edges of the rectangular loop has been meandered to guide the electromagnetic wave along the resonator so that a less insertion loss is found. In turn, the rectangular loop traps the electromagnetic wave for a while to feed the coupled-lines. It is noteworthy, the rectangular loop has been constructed with the same value of high impedance as the comb-lines. Such a construction improves the coupling effect for the whole design. The gap $(g)$ in Figure 1(a) determines the insertion loss level. Although the level of the insertion loss is decreased by decreasing the gap $(g)$, the level of the return loss also increases in this case. Thus, an optimized value for the gap has been chosen to design the resonator. Whereas, lengths of $l_{1}, l_{2}, l_{3}$ and $l_{4}$ determine the frequency of resonance. Because these lengths are equal for this design, perturbation of the resonant frequency can be performed by changing the lengths with the same increment or decrement.

To feed the resonator from the two ports, microstrip lines of $50 \Omega$ impedance have been utilized. Generally, for feed-lines, the length of microstrip lines is of a quarter-wave length. It is a useful technique to avoid erroneous impedance-matching. However, in this design, feed-lines are much shorter than the quarterwave length by employing a technique of connecting the resonator with the feed-lines via the tapered lines. Such a technique can remarkably elminate the dependency of quarter-wave length for the feed-lines. As a result, this technique is useful to miniaturize the design. In Figure 1(b), a lossless equivalent circuit has been illustrated to replace the microstrip impedance. Typically, the series combination of the total inductance and capacitance of any microstrip line represents its impedance since the microstrip lines are of distributed type.

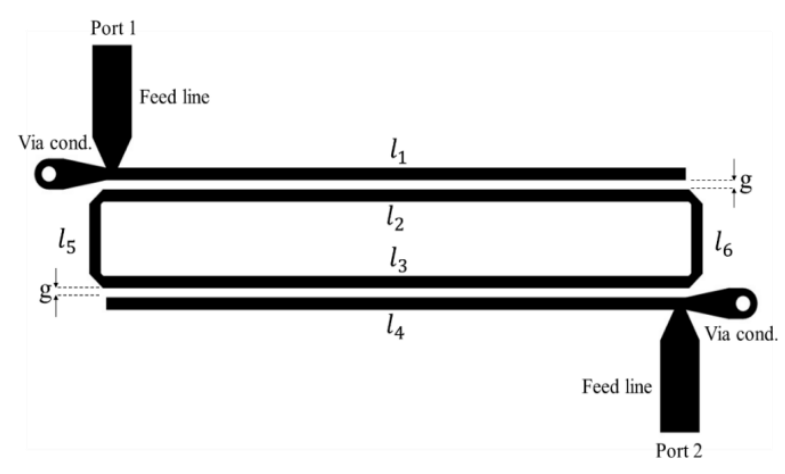

(a)

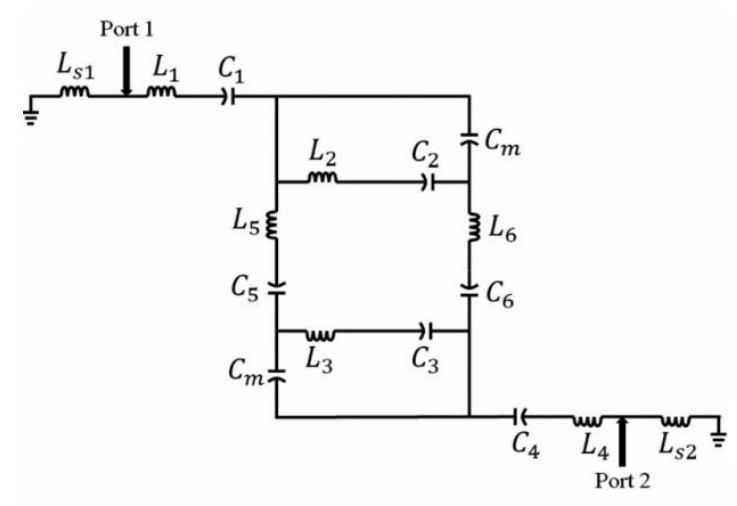

(b)

Figure 1. Proposed resonato,r (a) layout view, (b) lossless equivalent circuit [16] 
The equivalent $L C$-tank that represents the distributed impedance of the microstrip line. Therefore, the total equivalent inductance and the capacitance determine the center frequency $\left(f_{0}\right)$ for the resonant mode. For a particular microstrip resonator, the total inductance and the total capacitance determine the $f_{0}$ as follow.

$$
f_{0}=\frac{1}{2 \pi \sqrt{L C}}
$$

In this equation, for the microstrip resonator, the total inductance is denoted by $L$ and the total capacitance is denoted by $C$. As a result, each stub is represented by its series equivalent of total $L$ and $C$. For example, the short-circuited tapered line at port 1 in Figure 1(a) has been represented by its total inductance $L_{s 1}$ because a short-circuited stub works as an inductive path to the ground plane. In Figure 1(b), $C_{m}$ works as a parallel capacitor that represents the mutual capacitance because of the electric coupling between the comb-lines and rectangular loop. Because the equivalent circuit in Figure 1(b) characterizes the whole resonator construction, a total inductance and capacitance can be obtained from the equivalent circuit so that the resonant mode is found. Table 1 provides the corresponding dimensions of the hairpin resonator to construct its layout.

Table 1. Dimensions of the resonator

\begin{tabular}{ccc}
\hline Name & Length $(\mathrm{mm})$ & Width $(\mathrm{mm})$ \\
\hline $\boldsymbol{l}_{\mathbf{1}}$ & 20.2 & 0.4 \\
$\boldsymbol{l}_{\mathbf{2}}$ & 20.2 & 0.4 \\
$\boldsymbol{l}_{\mathbf{3}}$ & 20.2 & 0.4 \\
$\boldsymbol{l}_{\mathbf{4}}$ & 20.2 & 0.4 \\
$\boldsymbol{l}_{\mathbf{5}}$ & 2.1 & 0.4 \\
$\boldsymbol{l}_{\mathbf{6}}$ & 2.1 & 0.4 \\
$\mathrm{~g}$ & 20.2 & 0.3 \\
\hline
\end{tabular}

\section{PERFORMANCE ANALYSIS}

The hairpin resonator has been designed and simulated with the Advanced Design System (ADS) 2017 and CST Microwave Studio 2017 CAD-softwares. When the layout of the hairpin resonator has been generated, an electromagnetic momentum (EM) simulation has been carried out to analyze the performance of the resonator. Figure 2 shows the S-parameter response of the hairpin resonator. It has been observed that in the WLAN bandwidth, the resonator has a second-ordered quasi-Chebyshev response with the center frequency at $2.45 \mathrm{GHz}$. The return is found as minimum as $-36.71 \mathrm{~dB}$. Whereas, the insertion loss of the hairpin resonator has been decreased with the maximally flat response with a minimum value of $-0.36 \mathrm{~dB}$.

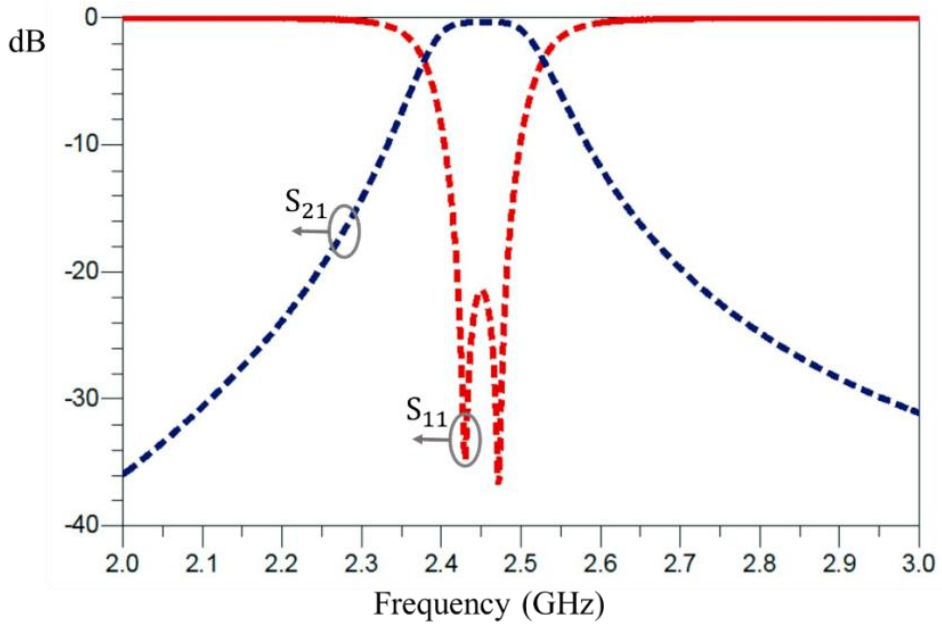

Figure 2. S-parameter response of the filter in WLAN frequency region 
As mentioned earlier, the resonator has a maximally flat response for the insertion loss, it exibits a better insertion loss than $-0.50 \mathrm{~dB}$ by covering a $70 \mathrm{MHz}$ bandwith $(2.42 \sim 2.49 \mathrm{GHz})$. In addition, the level of insertion loss is not worse than $-1 \mathrm{~dB}$ for the entire $100 \mathrm{MHz}$ WLAN bandwidth apart from the sharp roll-off factor of the resonator. This clearly indicates the ability of the hairpin resonator to introduce a high selectivity of the WLAN bandwidth with an improved transmission feature. However, the proposed hairpin resonator has a factional bandwidth (FBW) of $5.37 \%$.

Figure 3 indicates the surface current density and the quality factor of the hairpin resonator. For the non-resonant mode in Figure 3(a), if the electromagnetic signal passes from one port to another, a very small amount of surface current is accumulated near the transmitting port. This refers that the resonator rejects the electromagnetic signal to travel with a full transmission from one port to the other at the non-resonant mode.

Whereas according to Figure 3(b), at the resonant mode, a very high current density is found at the both comb-lines of the hairpin resonator. In fact, the surface current density obtains its highest value over one comb-line first and then decreases with the change of phase until another comb-line receives the same highest value. Such a phenomenon continues to happen throughout the resonant mode since the electromagnetic signal is trapped inside the hairpin resonator for a moment by employing the combination of a magnetic and an electric coupling effects of short-circuited comb-lines. Such a technique also improves the group-delay peak-based quality factor $(Q)$ of the hairpin resonator whereas $Q=\pi *$ frequency*(transmission group-delay).

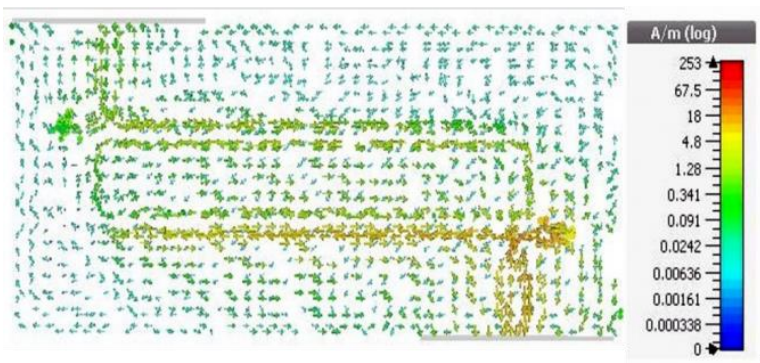

(a)

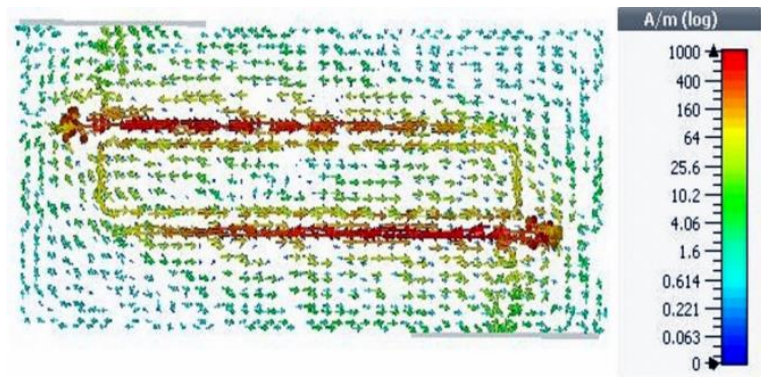

(b)

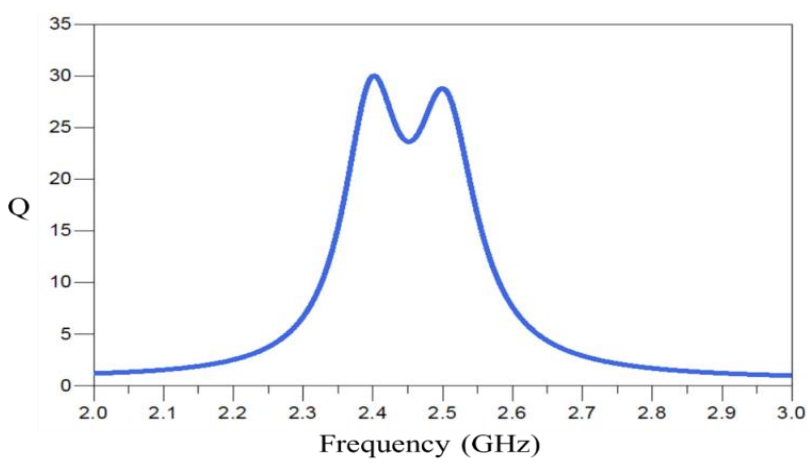

(c)

Figure 3. Hairpin resonator performance, (a) surface current density at the non-resonant mode,

(b) surface current density at the resonant mode, (c) group-delay peak-based quality factor

To implement the hairpin resonator on the Taconic TLX-8 substrate, the conventional etching process has been carried out. The comparison between the simulated and measured results has been presented in Figure 4. Due to the overreaction of the etching process in a laboratory scale and imperfect soldering of the ports, there has been a noticeable increase of insertion loss in the measured results. However, more agreement between the simulated and measured results is expected by an accurate implementation of the proposed resonator with the industrial process. Nevertheless, for a wideband response as in Figure 4, two transmission zeros at the both ends of the passband ensure the filtering stability of the hairpin resonator on a practical basis. 


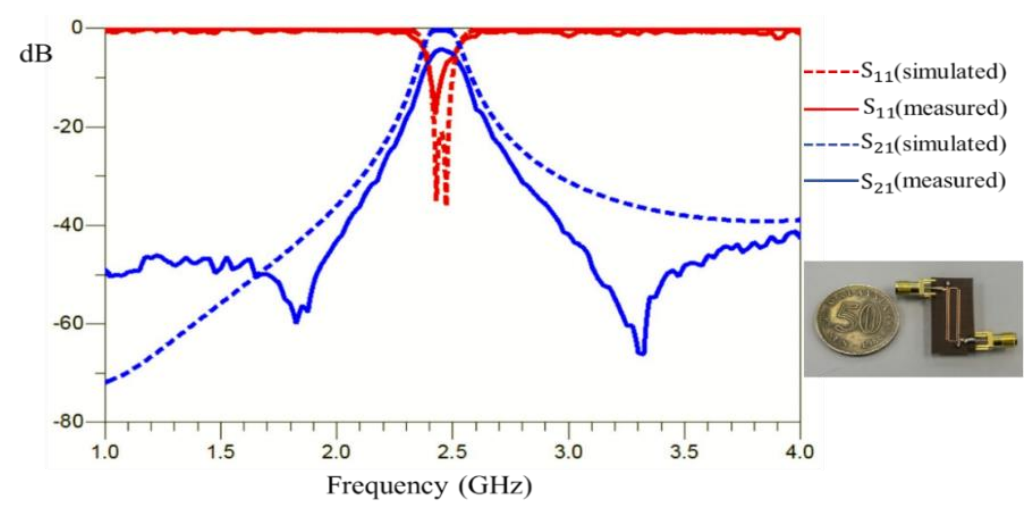

Figure 4. Wideband response of the implemented hairpin resonator (inset)

Within the same scope, the overall simulated performance of the hairpin resonator has been shown on Table 2 for a comparison with the simulated results of some resonators that are proposed very recently. Such a comparison indicates that the desinged hairpin resonator offers a high selectivity of WLAN bandwidth with a low insertion loss and small size.

Table 2. Performance comparison

\begin{tabular}{cccccll}
\hline Ref. & \multicolumn{6}{c}{ Resonator attributes } \\
& $\boldsymbol{f}_{\mathbf{0}}$ & FBW & $\begin{array}{c}\boldsymbol{S}_{\mathbf{2 1}} \\
(\mathrm{GHz})\end{array}$ & $\begin{array}{c}\boldsymbol{S}_{\mathbf{1 1}} \\
(\mathrm{dB})\end{array}$ & $\begin{array}{c}\text { Area } \\
\left(\mathrm{mm}^{2}\right)\end{array}$ & Substrate \\
\hline$[5]$ & 2.400 & 9.16 & -0.47 & -26.00 & 569.6 & RO4003C \\
{$[6]$} & 2.426 & 13.81 & -0.56 & -40.00 & 507.8 & F4B \\
{$[7]$} & 2.410 & 5.80 & -0.67 & -40.00 & 384.0 & RO4003 \\
{$[8]$} & 2.400 & 4.83 & -0.90 & -19.50 & 154.0 & RT/duroid \\
{$[9]$} & 2.420 & 6.60 & -0.40 & -36.00 & 3800.0 & RO4003 \\
{$[10]$} & 2.400 & 5.80 & -2.30 & -19.00 & 110.5 & RO4003 \\
{$[11]$} & 2.450 & 16.40 & -1.91 & -18.00 & 1520.0 & FR4 \\
{$[12]$} & 2.430 & 15.31 & -0.80 & -27.50 & 174.8 & RT/Duroid \\
{$[13]$} & 2.400 & 8.33 & -0.65 & -37.00 & 600.0 & FR4 \\
{$[14]$} & 2.400 & 3.50 & -0.50 & -30.00 & 325.1 & RT/Duroid \\
{$[15]$} & 2.400 & 9.17 & -2.07 & -14.93 & 625.0 & FR4 \\
This & 2.450 & 5.37 & -0.36 & -36.71 & 166.82 & Taconic \\
work & & & & & & TLX-8 \\
\hline
\end{tabular}

\section{CONCLUSION}

A miniaturized hairpin resonator has been designed and implemented at a center frequency of 2.45 GHz. The resonator sharply selects the $100 \mathrm{MHz}$ WLAN bandwidth with a low insertion loss. The effect of the electrical coupling between the rectangular loop and the short-circuited comb-lines plays a significant role to introduce a quasi-Chebyshev response by providing a maximally flat level of the insertion loss over the WLAN passband. A high attenuation over the stopband is also obtained so that the unwanted out-band signal is tightly eliminated. The proposed hairpin resonator is symmetrical and miniaturized in dimension which is less costly and cumbersome to implement for WLAN applications. Due to the increase of the groupdelay peak-based quality factor, this resonator is expected to be used further in the low-noise radio frequency integrated circuits (RFICs) applications.

\section{ACKNOWLEDGEMENTS}

This research has been supported by the Research Management Centre, International Islamic University Malaysia under the project of RIGS17-006-0581. 


\section{REFERENCES}

[1] S. M. K. Azam, M. I. Ibrahimy, S. M. A. Motakabber and A. K. M. Z. Hossain, "Microstrip Coupled Line Bandpass Filter with Radial Stubs for Narrow-Band Applications," Intl. J. GEOMATE, vol. 13, no. 40, pp. 183-188, Dec. 2017.

[2] S. M. K. Azam, M. I. Ibrahimy and S. M. A. Motakabber, "Reduction of phase noise for inductor based ultra-wide band voltage controlled oscillator," in Int. Conf. Commun., Control, Computing and Electronics Engineering (ICCCCEE), Khartoum, Jan. 2017, pp. 1-4.

[3] M. S. Islam, M. I. Ibrahimy, S. M. A. Motakabber and A. K. M. Z. Hossain, "A Rectangular Inset-Fed Patch Antenna with Defected Ground Structure for ISM Band," in Proc. 7th Intl. Conf. Computer and Communication Engineering (ICCCE), Kuala Lumpur, 2018, pp. 104-108.

[4] A. K. M. Z. Hossain, S. M. A. Motakabber and M. I. Ibrahimy. "Microstrip Spiral Resonator for the UWB Chipless RFID Tag.” In Progress in Systems Engineering, Cham, Springer, pp. 355-358, 2015.

[5] I. Islam, N. Islam and M. Haque, "A miniaturized interdigital hairpin microstrip bandpass filter design," in Intl. Conf. Informatics, Electronics and Vision (ICIEV), Dhaka, May. 2013, pp. 1-4.

[6] F. Cheng, X. Lin, P. Zhang, K. Song, and Y. Fan, "A microstrip bandpass filter based on inductive coupled quarterwavelength resonators," Microw. Optical Technol. Lett., vol. 55, no. 5, pp. 1031-1033, May. 2013.

[7] Q. Zhao, G. Wang, and D. Ding, "Compact microstrip bandpass filter with fragment-loaded resonators," Microw. Optical Technol. Lett., vol. 56, no. 12, pp. 2896-2899, Dec. 2014.

[8] K. Song, F. Zhang, and Y. Fan, "Miniaturized dual-band bandpass filter with good frequency selectivity using SIR and DGS," AEU-Intl. J. Electron. Commun., vol. 68, no. 5, pp. 384-387, May 2014.

[9] H. Wang, K.-W. Tam, S.-K. Ho, W. Kang, and W. Wu, "Short-Ended Self-Coupled Ring Resonator and Its Application for Balanced Filter Design," IEEE Microw. Wireless Compon. Lett., vol. 24, no. 5, pp. 312-314, May 2014.

[10] S. Xu, K. Ma, F. Meng, and K. S. Yeo, "Novel Defected Ground Structure and Two-Side Loading Scheme for Miniaturized Dual-Band SIW Bandpass Filter Designs," IEEE Microw. Wireless Compon. Lett., vol. 25, no. 4, pp. 217-219, Apr. 2015.

[11] S. Mora, Y. Alonso, N. Vargas, J. Vera, and J. Avendano, "Design of a bandpass filter using microstrip Hairpin resonators,"in CHILEAN Conf. Electrical, Electronics Engineering, Information and Communication Technologies (CHILECON), Oct. 2017, pp. 1-5.

[12] J. W. Eun and J.-H. Lee, "A microstrip dual-band bandpass filter using feed line with SIR," IEICE Electron. Express, vol. 14, no. 4, pp. 20170022-20170022, 2017.

[13] C. Kim, T. H. Lee, B. Shrestha, and K. C. Son, "Miniaturized dual-band bandpass filter based on stepped impedance resonators," Microw. Optical Technol. Lett., vol. 59, no. 5, pp. 1116-1119, May 2017.

[14] H.-W. Deng, F. Liu, T. Xu, L. Sun, and Y.-F. Xue, "Compact and high selectivity dual-mode microstrip BPF with frequency-dependent source-load coupling," Electron. Lett., vol. 54, no. 4, pp. 219-221, Jan. 2018.

[15] A. R. Othman and A. Ahmad, "Design and optimisation of narrow dual bandpass filter using bell-shaped structure for RF receiver system,” Intl. J. Wireless and Mobile Computing, vol. 14, no. 1, pp. 64-69, 2018.

[16] S. M. K. Azam, M. I. Ibrahimy, S. M. A. Motakabber and A. K. M. Zakir Hossain, "A Compact Bandpass Filter Using Microstrip Hairpin Resonator for WLAN Applications," in Proc. 7th Intl. Conf. on Computer and Communication Engineering (ICCCE), Kuala Lumpur, Sep. 2018, pp. 313-316.

[17] S. Azizi, M. E. Halaoui, A. Kaabal, S. Ahyoud and A. Asselman, "Enhanced Bandwidth of Band Pass Filter Using a Defected Microstrip Structure for Wideband Applications," Intl. J. Electrical and Computer Engineering (IJECE), vol. 8, no. 6, pp. 5260-5267, Dec. 2018. 